\title{
Propagation and nonlinear generation dynamics in a coherently prepared four-level system
}

\author{
E. Paspalakis, ${ }^{1}$ N. J. Kylstra, ${ }^{2}$ and P. L. Knight ${ }^{3}$ \\ ${ }^{1}$ Materials Science Department, School of Natural Sciences, University of Patras, Patras 265 04, Greece \\ ${ }^{2}$ Department of Physics, University of Durham, Durham DH1 3LE, United Kingdom \\ ${ }^{3}$ QOLS, Blackett Laboratory, Imperial College, London SW7 2BW, United Kingdom
}

(Received 18 December 2001; published 24 April 2002)

\begin{abstract}
We explore the laser-pulse propagation dynamics when one, two, or three pulses resonantly drive a coherently prepared four-level medium in a tripod configuration. If the system is prepared in a coherent superposition of two of the lower levels, we show that two incident resonant pulses can parametrically generate a third pulse in the medium. Over short distances, the propagation of this generated pulse can be partially controlled by varying the Rabi frequency of one of the driving fields. For a medium prepared in a coherent superposition of the three lower levels, we obtain a general expression for a three-photon Beer's length. Under specific conditions, the medium can either become completely opaque or completely transparent to three incident resonant pulses. Moreover, a single incident pulse can be used to parametrically generate two additional pulses. Finally, a general relationship is found that links the amplitudes of the initial superposition of states and the amplitudes of the pulses at large distances in the medium, implying that the initial superposition can be used to engineer the amplitudes of the exiting pulses.
\end{abstract}

DOI: $10.1103 /$ PhysRevA.65.053808

PACS number(s): 42.50.Gy, 42.50.Md

\section{INTRODUCTION}

Investigations of the propagation dynamics of laser pulses interacting with coherently prepared, resonantly driven multilevel media has revealed a number of interesting effects [1-4]. Examples include theoretical predictions of the enhancement of the index of refraction [5], the creation of matched pulses in thick media [6-12], and enhanced nonlinear pulse generation [13-17]. Experimental studies have also been carried out [18-25], demonstrating the potential for applications of these phenomena. In this paper, we analyze the propagation dynamics of three laser pulses in a coherently prepared medium, with the pulses resonantly coupling four levels of the system in a tripod configuration. This tripod system has been studied in detail with regard to targeted population transfer and the creation of tailored atomic superpositions [26,27]. In addition, it has been proposed as a robust system for generating nonabelian phases [28] and for qubit rotation [29]. Experimental results showing coherence switching [30] with applications in subpicosecond optical switching [31] have also been presented.

We have recently demonstrated that double transparency and parametric generation can occur in this system when continuous wave (cw) laser fields are used [32]. In this paper, we continue our study by showing that similar results can be obtained when the tripod system is driven by short laser pulses. The paper is organized as follows. In the next section we present the main equations that govern the propagation dynamics of laser pulses in our system. In Sec. III, by assuming that the laser-matter interaction is weak, we obtain an analytical solution for the Rabi frequencies of the system. We show that, if the system is initially prepared in two of the lower states, the propagation and nonlinear generation dynamics of the system can be partially controlled by the laser field which couples the initially unoccupied lower state to the upper state. Our analytical results for controllable matched pulse propagation and parametric generation are also com- pared with numerical results. In Sec. IV, we assume that the system is prepared in a coherent superposition of all of the lower levels and solve the coupled Maxwell-Schrödinger equations, again for the case of weak laser-matter coupling. A three-photon Beer's law that governs the propagation dynamics of the laser pulses in this medium is then defined. In addition, we show that two laser pulses can be generated in this medium by initially injecting one laser pulse. The analytical results for pulse propagation and nonlinear generation are compared with results obtained by numerically solving the Maxwell-Schrödinger equation. We continue in Sec. V by deriving a general propagation relation which holds for large distances in a coherently prepared tripod system, irrespective of the strength of the laser-matter interaction. In particular, the laser pulses reshape according to a general algebraic relation that connects the amplitudes of the initial coherent superposition and the Rabi frequencies. The validity of this relation is verified by numerical simulations. Our findings are summarized in Sec. VI.

\section{THEORETICAL MODEL}

The quantum system under consideration is shown in Fig. 1. The excited state $|3\rangle$ is coupled by three coherent laser pulses to the lower levels $|0\rangle,|1\rangle$, and $|2\rangle$. The interaction Hamiltonian of this system, in the rotating wave and dipole approximations, is given by $(\hbar=1)$

$$
\begin{aligned}
H= & \Omega_{0} e^{-i \delta_{0} t-i k_{0} z}|0\rangle\left\langle 3\left|+\Omega_{1} e^{-i \delta_{1} t-i k_{1} z}\right| 1\right\rangle\langle 3| \\
& +\Omega_{2} e^{-i \delta_{2} t-i k_{2} z}|2\rangle\langle 3|+\text { H.c. }
\end{aligned}
$$

Here, $\Omega_{n}(\zeta, \tau)=-\vec{\mu}_{n 3} \cdot \hat{\varepsilon}_{n} \mathcal{E}_{n} f_{n}(\zeta, \tau), \quad n=0,1,2$, are the Rabi frequencies and $\delta_{n}=\omega_{3 n}-\omega_{n}$ are the laser field detunings from resonance. The quantities $\vec{\mu}_{n 3}$ and $\omega_{3 n}$, respectively, are the dipole matrix element and angular frequency 


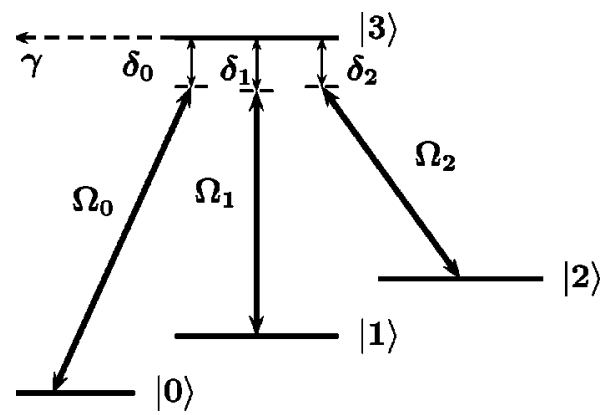

FIG. 1. Schematic diagram of the system studied. It consists of three lower levels $(|0\rangle,|1\rangle$, and $|2\rangle)$ and a single upper level $|3\rangle$ that decays out of the system. The lower states are coupled near resonantly to the excited state by three laser pulses.

of the $|n\rangle \leftrightarrow|3\rangle$ transition. The laser field is described classically as a time- and spatially-dependent electric field

$$
\vec{E}(z, t)=\sum_{n=0}^{2} \hat{\varepsilon}_{n}\left[\mathcal{E}_{n} f_{n}(z, t) e^{i\left(\omega_{n} t-k_{n} z\right)}+\text { c.c. }\right],
$$

where $\omega_{n}$ is the angular frequency, $k_{n}$ is the wave number, $\hat{\varepsilon}_{n}$ is the polarization vector which is perpendicular to the propagation direction $\hat{z}, \mathcal{E}_{n}$ is the electric-field amplitude, and $f_{n}(z, t)$ is the dimensionless pulse envelope of the laser field.

The system can be described by a state vector that is expanded in terms of the atomic states multiplied by the corresponding space and time-dependent amplitudes $b_{n}(z, t)$ as

$$
\begin{aligned}
|\psi(z, t)\rangle= & b_{0}(z, t)|0\rangle+b_{1}(z, t) e^{i\left(\delta_{0}-\delta_{1}\right) t+i\left(k_{0}-k_{1}\right) z}|1\rangle \\
& +b_{2}(z, t) e^{i\left(\delta_{0}-\delta_{2}\right) t+i\left(k_{0}-k_{2}\right) z}|2\rangle \\
& +b_{3}(z, t) e^{i \delta_{0} t+i k_{0} z}|3\rangle .
\end{aligned}
$$

We substitute Eqs. (1)-(3) into the Schrödinger equation to obtain,

$$
i \frac{\partial}{\partial t} \mathbf{b}(z, t)=\mathbf{H}(z, t) \mathbf{b}(z, t)
$$

with

$$
\mathbf{H}(z, t)=\left(\begin{array}{cccc}
0 & 0 & 0 & \Omega_{0}(z, t) \\
0 & \delta_{0}-\delta_{1} & 0 & \Omega_{1}(z, t) \\
0 & 0 & \delta_{0}-\delta_{2} & \Omega_{2}(z, t) \\
\Omega_{0}^{*}(z, t) & \Omega_{1}^{*}(z, t) & \Omega_{2}^{*}(z, t) & \left(\delta_{0}-i \frac{\gamma}{2}\right)
\end{array}\right)
$$

and $\mathbf{b}(z, t)=\left[b_{0}(z, t), b_{1}(z, t), b_{2}(z, t), b_{3}(z, t)\right]^{T}$. Here, $\gamma$ denotes the decay rate of the excited state out of the system.

In order to study the propagation of short laser pulses in this medium, the Maxwell wave equation is required, which in the slowly varying envelope approximation reads

$$
\begin{aligned}
& {\left[\frac{\partial}{\partial z} f_{n}(z, t)+\frac{1}{c} \frac{\partial}{\partial t} f_{n}(z, t)\right] \hat{\varepsilon}_{n} \mathcal{E}_{n} e^{i\left(\omega_{n} t-k_{n} z\right)}} \\
& \quad=-\frac{2 i \pi \omega_{n}}{c} \vec{P}_{n}(z, t)
\end{aligned}
$$

If dephasing processes such as Doppler broadening are ignored, the negative frequency part of the macroscopic polarization of the medium, $\vec{P}_{n}(z, t)$ is given by

$$
\vec{P}_{n}(z, t)=\mathcal{N} \vec{\mu}_{3 n} b_{n}(z, t) b_{3}^{*}(z, t) e^{i \omega_{n} t-i k_{n} z} .
$$

Substituting Eq. (7) into Eq. (6), transforming the resulting equation to the local (retarded) frame where $\tau=t-z / c$ and $\zeta=z$, and using Eq. (4) we obtain the coupled MaxwellSchrödinger equations that govern the evolution of the system,

$$
\begin{gathered}
i \frac{\partial}{\partial \tau} \mathbf{b}(\zeta, \tau)=\mathbf{H}(\zeta, \tau) \mathbf{b}(\zeta, \tau), \\
\frac{\partial}{\partial \zeta} \Omega_{n}(\zeta, \tau)=i a_{n} b_{n}(\zeta, \tau) b_{3}^{*}(\zeta, \tau), \quad n=0,1,2 .
\end{gathered}
$$

Here, $a_{n}=2 \pi \mathcal{N}\left|\vec{\mu}_{n 3}\right|^{2} \omega_{n} / c$ is the propagation constant.

\section{CONTROL OF PULSE PROPAGATION AND GENERATION}

In this section we assume that the system is initially prepared in a superposition of two of the lower levels, say $|0\rangle$ and $|1\rangle$, such that

$$
|\psi(\zeta, \tau=0)\rangle=\alpha|0\rangle+\beta|1\rangle,
$$

with $\alpha$ and $\beta$ being, in general, imaginary constants satisfying $|\alpha|^{2}+|\beta|^{2}=1$. We also assume that the two-photon resonance condition, $\delta_{0}=\delta_{1}=\delta$, is satisfied. If the medium is heavily damped and the laser-matter interaction is weak, the approximate solution of Eq. (8) is $[3,8,9]$

$$
\begin{gathered}
b_{0}(\zeta, \tau) \approx \alpha, \quad b_{1}(\zeta, \tau) \approx \beta, \\
b_{2}(\zeta, \tau) \approx-\frac{\Omega_{2}(\zeta=0, \tau) b_{3}(\zeta, \tau)}{\delta-\delta_{2}}, \\
b_{3}(\zeta, \tau)=-\frac{\Omega_{0}^{*}(\zeta, \tau) \alpha+\Omega_{1}^{*}(\zeta, \tau) \beta}{\delta-\left|\Omega_{2}(\zeta=0, \tau)\right|^{2} /\left(\delta-\delta_{2}\right)-i \gamma / 2},
\end{gathered}
$$

and Eqs. (9) reduce to

$$
\frac{\partial}{\partial \zeta} \boldsymbol{\Omega}(\zeta, \tau)=-i \mathbf{K}(\zeta, \tau) \boldsymbol{\Omega}(\zeta, \tau),
$$

where

$$
\mathbf{K}(\zeta, \tau)=\left(\begin{array}{cc}
\kappa_{0}(\tau)|\alpha|^{2} & \kappa_{0}(\tau) \alpha \beta^{*} \\
\kappa_{1}(\tau) \alpha^{*} \beta & \kappa_{1}(\tau)|\beta|^{2}
\end{array}\right)
$$


Here, $\boldsymbol{\Omega}(\zeta, \tau)=\left[\Omega_{0}(\zeta, \tau), \Omega_{1}(\zeta, \tau)\right]^{T}$ and $\kappa_{n}(\tau)=a_{n} /[\delta$ $\left.-\left|\Omega_{2}(\zeta=0, \tau)\right|^{2} /\left(\delta-\delta_{2}\right)+i \gamma / 2\right]$, with $n=0,1$.

With the initial conditions $\Omega_{0}(\zeta=0, \tau)=\bar{\Omega}_{0}(\tau)$ and $\Omega_{1}(\zeta=0, \tau)=\bar{\Omega}_{1}(\tau)$, the solution of Eq. (12) is then

$$
\begin{aligned}
\Omega_{0}(\zeta, \tau)= & \frac{1}{|\alpha|^{2} \kappa_{0}(\tau)+|\beta|^{2} \kappa_{1}(\tau)} \\
& \times\left[\left(|\alpha|^{2} \kappa_{0}(\tau) e^{-i\left[|\alpha|^{2} \kappa_{0}(\tau)+|\beta|^{2} \kappa_{1}(\tau)\right] \zeta}\right.\right. \\
& \left.+|\beta|^{2} \kappa_{1}(\tau)\right) \bar{\Omega}_{0}(\tau)+\alpha \beta^{*} \kappa_{0}(\tau) \\
& \left.\times\left(-1+e^{-i\left[|\alpha|^{2} \kappa_{0}(\tau)+|\beta|^{2} \kappa_{1}(\tau)\right] \zeta}\right) \bar{\Omega}_{1}(\tau)\right],
\end{aligned}
$$

$$
\begin{aligned}
\Omega_{1}(\zeta, \tau)= & \frac{1}{|\alpha|^{2} \kappa_{0}(\tau)+|\beta|^{2} \kappa_{1}(\tau)}\left[\alpha^{*} \beta \kappa_{1}(\tau)\right. \\
& \times\left(-1+e^{-i\left[|\alpha|^{2} \kappa_{0}(\tau)+|\beta|^{2} \kappa_{1}(\tau)\right] \zeta}\right) \bar{\Omega}_{0}(\tau) \\
& +\left(|\beta|^{2} \kappa_{1}(\tau) e^{-i\left[|\alpha|^{2} \kappa_{0}(\tau)+|\beta|^{2} \kappa_{1}(\tau)\right] \zeta}\right. \\
& \left.\left.+|\alpha|^{2} \kappa_{0}(\tau)\right) \bar{\Omega}_{1}(\tau)\right] .
\end{aligned}
$$

From these two equations it is seen that the spatial evolution of the pulses depends on the initial populations, $|\alpha|^{2}$ and $|\beta|^{2}$, as well as the initial coherence $\alpha^{*} \beta$. We also note that the two pulses reach a steady-state value at a rate that is determined by the two-photon Beer's length (see Refs. [8,9]) $\bar{\zeta}(\tau)^{-1}=-2 \operatorname{Im}\left[|\alpha|^{2} \kappa_{0}(\tau)+|\beta|^{2} \kappa_{1}(\tau)\right]$, which is obtained from the nonzero eigenvalue of the propagation matrix. Two interesting cases are readily obtained from Eqs. (14) and (15) when pulses with equal initial Rabi frequencies, $\bar{\Omega}_{0}(\tau)$ $=\bar{\Omega}_{1}(\tau)=\Omega(\tau)$, are applied to the medium. First, setting $\alpha a_{0}=\beta a_{1}$ we obtain that $\Omega_{0}(\zeta \rightarrow \infty, \tau)=\Omega_{1}(\zeta \rightarrow \infty, \tau)=0$, i.e., both pulses are completely absorbed by the medium. However, if $\alpha=-\beta$ then $\Omega_{0}(\zeta, \tau)=\Omega(\tau)$ and $\Omega_{1}(\zeta, \tau)$ $=\Omega(\tau)$, so that the medium is transparent to the pulses and they propagate as in free space.

Let us now discuss the possibility of parametric generation in our model. If $\bar{\Omega}_{0}(\tau)=\bar{\Omega}(\tau)$ and $\bar{\Omega}_{1}(\tau)=0$, Eqs. (14) and (15) reduce to

$$
\begin{aligned}
\Omega_{0}(\zeta, \tau)= & \frac{1}{|\alpha|^{2} \kappa_{0}(\tau)+|\beta|^{2} \kappa_{1}(\tau)} \\
& \left(|\alpha|^{2} \kappa_{0}(\tau) e^{-i\left[|\alpha|^{2} \kappa_{0}(\tau)+|\beta|^{2} \kappa_{1}(\tau)\right] \zeta}\right. \\
& \left.+|\beta|^{2} \kappa_{1}(\tau)\right) \bar{\Omega}(\tau) \\
\Omega_{1}(\zeta, \tau)= & \frac{1}{|\alpha|^{2} \kappa_{0}(\tau)+|\beta|^{2} \kappa_{1}(\tau)} \alpha^{*} \beta \kappa_{1}(\tau) \\
& \times\left(-1+e^{-i\left[|\alpha|^{2} \kappa_{0}(\tau)+|\beta|^{2} \kappa_{1}(\tau)\right] \zeta}\right) \bar{\Omega}(\tau) .
\end{aligned}
$$

It is seen that, via coherent internal generation, a medium coherently prepared in two of the lower levels will generate an additional field. This will occur only if the initial state of the system is coherent, otherwise phase fluctuations will average out the generation process, as can be seen from the right-hand side of Eq. (17). In particular, the Rabi frequency of the generated pulse is proportional to the coherence of the initial state.

In order to facilitate further analysis of parametric generation, we continue to simplify the equations by assuming that the propagation constants are equal, $a_{1}=a_{2}=a$. This leads to $\kappa_{0}(\tau)=\kappa_{1}(\tau)=\kappa(\tau)$, and the pulses describing the parametric generation process, Eqs. (16) and (17), reduce to
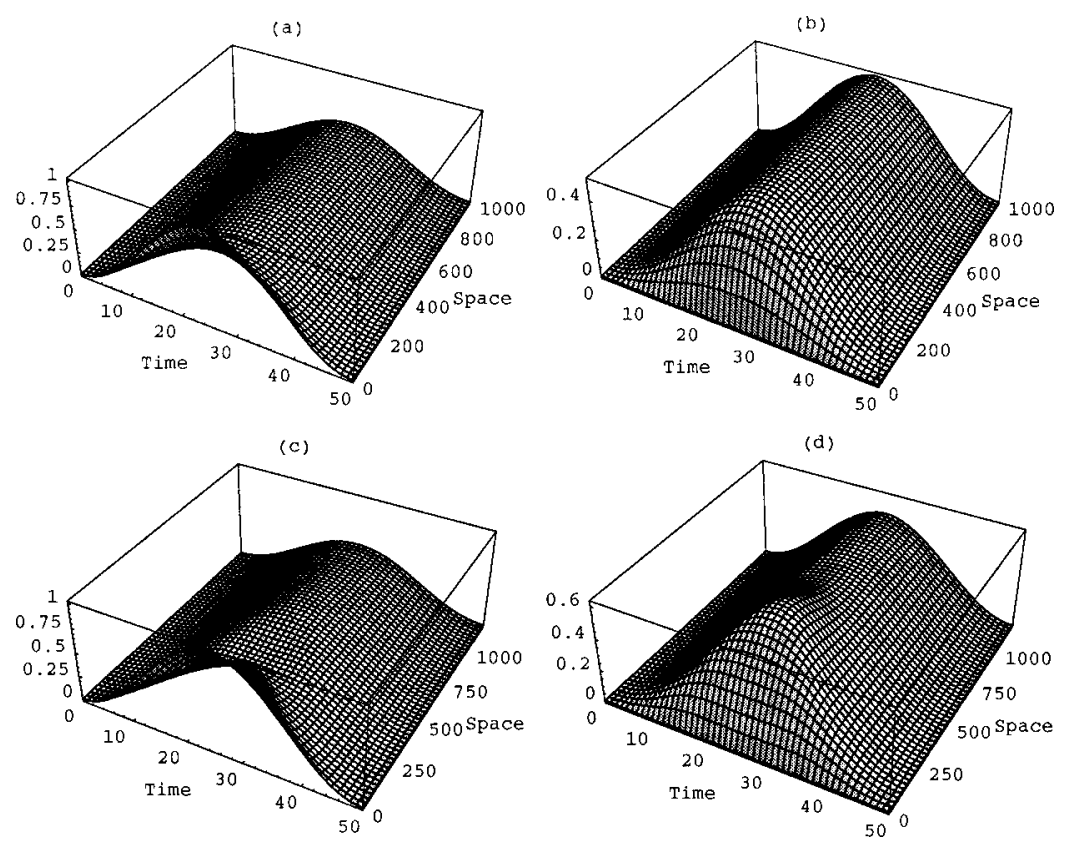

FIG. 2. Normalized field amplitudes $\left|\Omega_{0}(\zeta, \tau)\right| / \Omega_{0}$ (a) and (c); $\left|\Omega_{1}(\zeta, \tau)\right| / \Omega_{0}$ (b) and (d). The incident pulses are $\Omega_{0}(0, \tau)$ $=\Omega_{0} \sin ^{2}\left(\tau \pi / \tau_{p}\right), \quad \Omega_{1}(0, \tau)=0, \quad$ and $\quad \Omega_{2}(0, \tau)$ $=\Omega_{2} \sin ^{2}\left(\tau \pi / \tau_{p}\right)$, with $0 \leqslant \tau \leqslant \tau_{p}$. The system is initially in the superposition $|\psi(\zeta, 0)\rangle=1 / \sqrt{2}(|0\rangle$ $+|1\rangle$ ). In (a) and (b) $\Omega_{2}=5$, while in (c) and (d) $\Omega_{2}=10$. The remaining parameters used in the calculations are $a_{0}=a_{1}=a_{2}=1, \Omega_{0}=0.1, \tau_{p}$ $=50, \delta=1$, and $\gamma=100$. All quantities are in arbitrary units. 


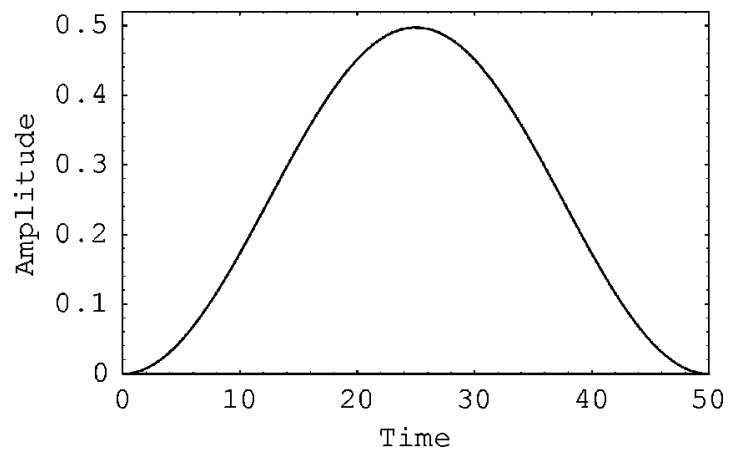

FIG. 3. Numerical and analytical normalized field amplitudes $\left|\Omega_{0}(\zeta, \tau)\right| / \Omega_{0}$ at position $\zeta=300$ in the medium. The numerical and analytical results cannot be distinguished in the figure. The medium and pulse parameters are as in Fig. 2. Similar agreement between the analytical and numerical results is obtained for $\left|\Omega_{1}(\zeta, \tau)\right| / \Omega_{0}$.

$$
\begin{aligned}
& \Omega_{0}(\zeta, \tau)=\left(|\alpha|^{2} e^{-i \kappa(\tau) \zeta}+|\beta|^{2}\right) \bar{\Omega}(\tau), \\
& \Omega_{1}(\zeta, \tau)=\alpha^{*} \beta\left(-1+e^{-i \kappa(\tau) \zeta}\right) \bar{\Omega}(\tau) .
\end{aligned}
$$

We note that the final (long-distance) result is independent of $\kappa(\tau), \quad$ as $\quad \Omega_{0}(\zeta \rightarrow \infty, \tau)=|\beta|^{2} \bar{\Omega}(\tau) \quad$ and $\quad \Omega_{1}(\zeta \rightarrow \infty, \tau)=$ $-\alpha^{*} \beta \bar{\Omega}(\tau)$. An important consequence of the scheme considered here, as compared to previously studied systems $[6-10,12-15,17]$, is that the propagation constants $\kappa_{0}(\tau)$, $\kappa_{1}(\tau)$ are functions of the third field, allowing the transient propagation dynamics of the system to be controlled by this field. This is illustrated in Fig. 2 where the time and space evolution of the laser pulses is shown, as obtained using the analytical results given by Eqs. (16) and (17), for two different values of the Rabi frequency of the control field (i.e., the field having angular frequency $\left.\omega_{2}\right)$. In the first case, Figs. 2(a) and 2(b), the spatial evolution of the fields is smooth. However, increasing the intensity of the control field [see Figs. 2(c) and 2(d)] will lead to an oscillation of the generated field. It should be noted that in both cases the generated pulses become the same at long distances in the medium. In addition, the initial and generated fields are matched [33], as can be seen from Eqs. (18) and (19).

The validity of the analytical results has been confirmed by comparing them with results that have been obtained by numerically solving Eqs. (8) and (9). These are shown in Fig. 3. It is clear that the agreement between the two results is very good, implying that our approximations are valid for the chosen set of parameters. We also note that matched pulses with envelopes quite different from the envelopes of the initially injecting pulses can be generated in this medium. This is demonstrated in Fig. 4 where two sin-squared pulses of different duration leads to a pair of doubly-peaked pulses in the medium.

\section{THREE-PHOTON BEER'S LAW AND PARAMETRIC GENERATION}

Let us now assume that the system is initially prepared in a coherent superposition of all the three lower levels, such that
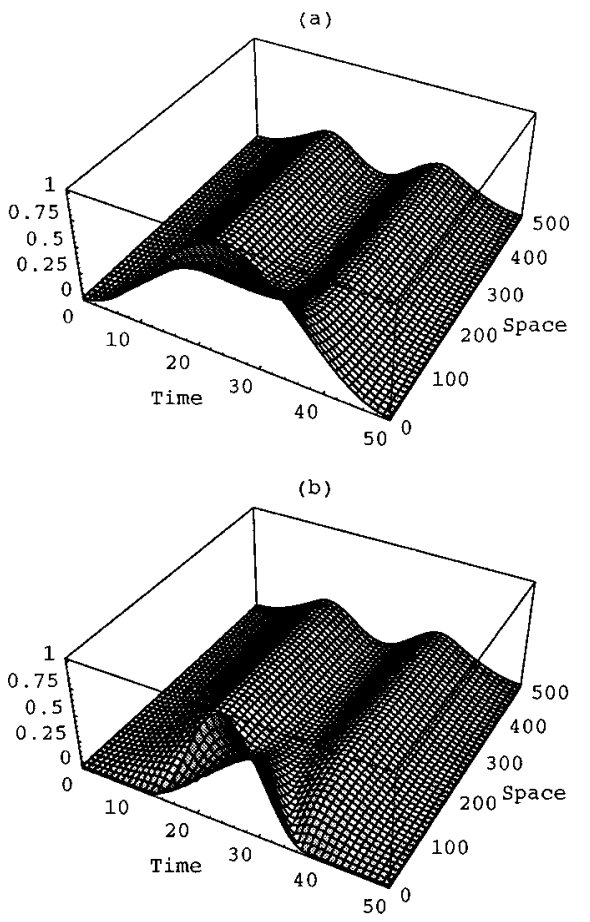

FIG. 4. Normalized field amplitudes $\left|\Omega_{0}(\zeta, \tau)\right| / \Omega_{0}$ (a) and $\left|\Omega_{1}(\zeta, \tau)\right| / \Omega_{0}$ (b), for initial pulses $\Omega_{0}(0, \tau)=\Omega_{0} \sin ^{2}\left(\tau \pi / \tau_{p}\right)$, $\Omega_{2}(0, \tau)=\Omega_{0} \sin ^{2}\left(\tau \pi / \tau_{p}\right) \quad\left(0 \leqslant \tau \leqslant \tau_{p}\right), \quad$ and $\quad \Omega_{1}(0, \tau)=\Omega_{1} \sin ^{2}[2(\tau$ $\left.\left.-\tau_{p} / 4\right) \pi / \tau_{p}\right] \quad\left(\tau_{p} / 4 \leqslant \tau \leqslant 3 \tau_{p} / 4\right)$. The system is initially in the superposition $|\psi(\zeta, 0)\rangle=1 / \sqrt{2}(|0\rangle+|1\rangle)$, and $\Omega_{2}=5$. The remaining medium and pulse parameters are the same as in Fig. 2.

$$
|\psi(0)\rangle=\alpha|0\rangle+\beta|1\rangle+\gamma|2\rangle
$$

with $\alpha, \beta$, and $\gamma$ being, in general, imaginary constants satisfying $|\alpha|^{2}+|\beta|^{2}+|\gamma|^{2}=1$. We also assume that the threephoton resonance condition $\delta_{0}=\delta_{1}=\delta_{2}=\delta$ holds. As in the previous section, we take the medium to be heavily damped and the laser-matter interaction to be weak. Under these conditions, the approximate solution of Eq. (8) is

$$
\begin{gathered}
b_{0}(\zeta, \tau) \approx \alpha, \quad b_{1}(\zeta, \tau) \approx \beta, \quad b_{2}(\zeta, \tau) \approx \gamma, \\
b_{3}(\zeta, \tau)=-\frac{\Omega_{0}^{*}(\zeta, \tau) \alpha+\Omega_{1}^{*}(\zeta, \tau) \beta+\Omega_{2}^{*}(\zeta, \tau) \gamma}{\delta-i \gamma / 2} .
\end{gathered}
$$

Then, Eqs. (9) become

$$
\frac{\partial}{\partial \zeta} \boldsymbol{\Omega}(\zeta, \tau)=-i \mathbf{K}(\zeta, \tau) \boldsymbol{\Omega}(\zeta, \tau)
$$

where

$$
\mathbf{K}(\zeta, \tau)=\left(\begin{array}{ccc}
\kappa_{0}^{\prime}|\alpha|^{2} & \kappa_{0}^{\prime} \alpha \beta^{*} & \kappa_{0}^{\prime} \alpha \gamma^{*} \\
\kappa_{1}^{\prime} \alpha^{*} \beta & \kappa_{1}^{\prime}|\beta|^{2} & \kappa_{1}^{\prime} \beta \gamma^{*} \\
\kappa_{2}^{\prime} \alpha^{*} \gamma & \kappa_{2}^{\prime} \beta^{*} \gamma & \kappa_{2}^{\prime}|\gamma|^{2}
\end{array}\right),
$$




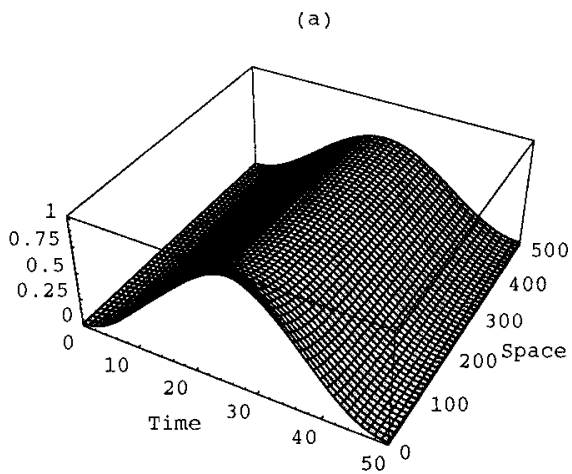

(b)

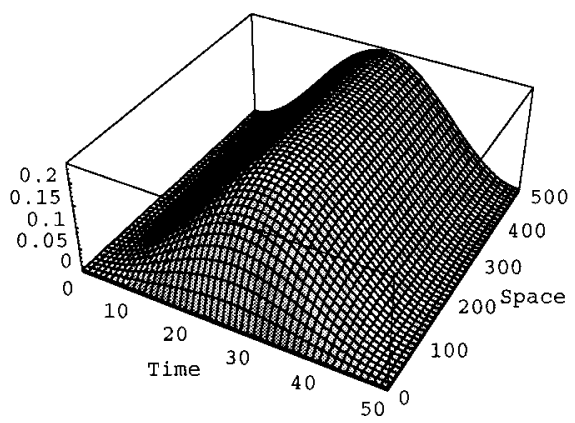

(c)

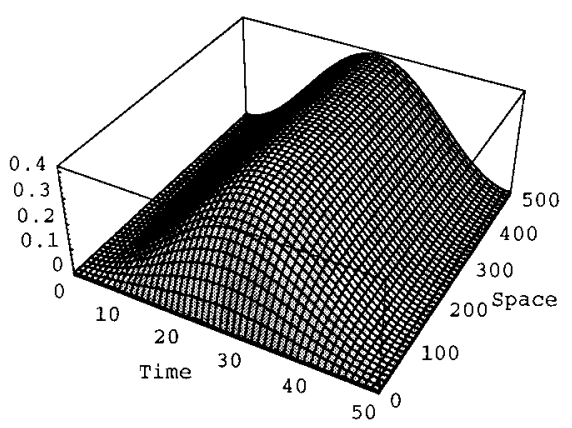

FIG. 5. Normalized field amplitudes $\left|\Omega_{0}(\zeta, \tau)\right| / \Omega_{0} \quad$ (a), $\left|\Omega_{1}(\zeta, \tau)\right| / \Omega_{0}$ (b), and $\left|\Omega_{2}(\zeta, \tau)\right| / \Omega_{0}$ (c), for an initial pulse $\Omega_{0}(0, \tau)=\Omega_{0} \sin ^{2}\left(\tau \pi / \tau_{p}\right)\left(0 \leqslant \tau \leqslant \tau_{p}\right) \quad$ and $\quad \Omega_{1}(0, \tau)=\Omega_{2}(0, \tau)=0$. The system is initially in the superposition $|\psi(\zeta, 0)\rangle=1 / \sqrt{3}|0\rangle$ $+1 / \sqrt{6}|1\rangle+1 / \sqrt{2}|2\rangle$. The two-photon resonant detuning is $\delta=0$. The remaining medium and pulse parameters are the same as in Fig. 2.

$\boldsymbol{\Omega}(\zeta, \tau)=\left[\Omega_{0}(\zeta, \tau), \Omega_{1}(\zeta, \tau), \Omega_{2}(\zeta, \tau)\right]^{T} \quad$ and $\quad \kappa_{n}^{\prime}=a_{n} /(\delta$ $+i \gamma / 2)$, with $n=0,1,2$.

The solution of Eq. (22) with initial conditions $\Omega_{0}(\zeta=0, \tau)=\bar{\Omega}_{0}(\tau), \quad \Omega_{1}(\zeta=0, \tau)=\bar{\Omega}_{1}(\tau), \quad \Omega_{2}(\zeta=0, \tau)$ $=\bar{\Omega}_{2}(\tau)$ is given by

$$
\begin{aligned}
\Omega_{0}(\zeta, \tau)= & \frac{1}{\lambda}\left[\left(|\alpha|^{2} \kappa_{0}^{\prime} e^{-i \lambda \zeta}+|\beta|^{2} \kappa_{1}^{\prime}+|\gamma|^{2} \kappa_{2}^{\prime}\right) \bar{\Omega}_{0}(\tau)\right. \\
& +\alpha \beta^{*} \kappa_{0}^{\prime}\left(-1+e^{-i \lambda \zeta}\right) \bar{\Omega}_{1}(\tau) \\
& \left.+\alpha \gamma^{*} \kappa_{0}^{\prime}\left(-1+e^{-i \lambda \zeta}\right) \bar{\Omega}_{2}(\tau)\right]
\end{aligned}
$$

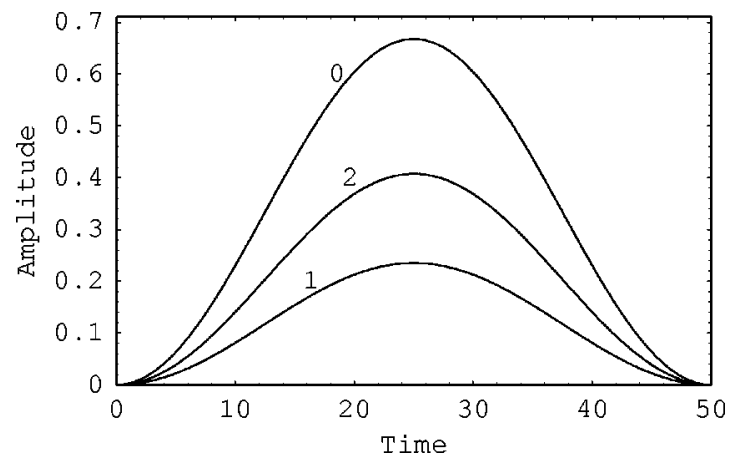

FIG. 6. As indicated, the numerical and analytical normalized field amplitudes $\left|\Omega_{0}(\zeta, \tau)\right| / \Omega_{0},\left|\Omega_{1}(\zeta, \tau)\right| / \Omega_{0}$, and $\left|\Omega_{2}(\zeta, \tau)\right| / \Omega_{0}$, at $\zeta=300$ in the medium for the same parameters as in Fig. 5. The numerical and analytical results are indistinguishable.

$$
\begin{aligned}
\Omega_{1}(\zeta, \tau)= & \frac{1}{\lambda}\left[\alpha^{*} \beta \kappa_{1}^{\prime}\left(-1+e^{-i \lambda \zeta}\right) \bar{\Omega}_{0}(\tau)\right. \\
& +\left(|\alpha|^{2} \kappa_{0}^{\prime}+|\beta|^{2} \kappa_{1}^{\prime} e^{-i \lambda \zeta}+|\gamma|^{2} \kappa_{2}^{\prime}\right) \bar{\Omega}_{1}(\tau) \\
& \left.+\beta \gamma^{*} \kappa_{1}^{\prime}\left(-1+e^{-i \lambda \zeta}\right) \bar{\Omega}_{2}(\tau)\right], \\
\Omega_{2}(\zeta, \tau)= & \frac{1}{\lambda}\left[\alpha^{*} \gamma \kappa_{2}^{\prime}\left(-1+e^{-i \lambda \zeta}\right) \bar{\Omega}_{0}(\tau)\right. \\
& +\beta^{*} \gamma \kappa_{2}^{\prime}\left(-1+e^{-i \lambda \zeta}\right) \bar{\Omega}_{1}(\tau)+\left(|\alpha|^{2} \kappa_{0}^{\prime}+|\beta|^{2} \kappa_{1}^{\prime}\right. \\
& \left.\left.+|\gamma|^{2} \kappa_{2}^{\prime} e^{-i \lambda \zeta}\right) \bar{\Omega}_{2}(\tau)\right],
\end{aligned}
$$

with $\lambda=|\alpha|^{2} \kappa_{0}^{\prime}+|\beta|^{2} \kappa_{1}^{\prime}+|\gamma|^{2} \kappa_{2}^{\prime}$. We note that the spatial evolution of the pulses again depends on the initial population and on the initial coherence of the system. We also note that the three pulses reach a steady-state value at a rate that is determined by the three-photon Beer's length

$$
\frac{1}{\bar{\zeta}}=-2 \operatorname{Im}(\lambda)
$$

whose value is obtained from the nonzero eigenvalue $\lambda$ of the propagation matrix. From Eqs. (24)-(26) we again can readily obtain conditions that lead to either complete absorption or transparency. Setting $\bar{\Omega}_{0}(\tau)=\bar{\Omega}_{1}(\tau)=\bar{\Omega}_{2}(\tau)$ $=\Omega(\tau)$, it is seen that when $\alpha a_{0}=\beta a_{1}=\gamma a_{2}$ then $\Omega_{0}(\zeta$ $\rightarrow \infty, \tau)=\Omega_{1}(\zeta \rightarrow \infty, \tau)=\Omega_{2}(\zeta \rightarrow \infty, \tau)=0$, i.e., the pulses are completely absorbed by the medium. However, if $\alpha+\beta+\gamma=0 \quad$ then $\Omega_{0}(\zeta, \tau)=\Omega(\tau), \quad \Omega_{1}(\zeta, \tau)=\Omega(\tau)$, $\Omega_{2}(\zeta, \tau)=\Omega(\tau)$, and the medium is transparent to the pulses.

Let us now consider the possibility of parametric generation. If $\bar{\Omega}_{0}(\tau)=\bar{\Omega}(\tau)$ and $\bar{\Omega}_{1}(\tau)=\bar{\Omega}_{2}(\tau)=0$ then from Eqs. (24)-(26) we have

$$
\Omega_{0}(\zeta, \tau)=\frac{1}{\lambda}\left(|\alpha|^{2} \kappa_{0}^{\prime} e^{-i \lambda \zeta}+|\beta|^{2} \kappa_{1}^{\prime}+|\gamma|^{2} \kappa_{2}^{\prime}\right) \bar{\Omega}(\tau),
$$


(a)

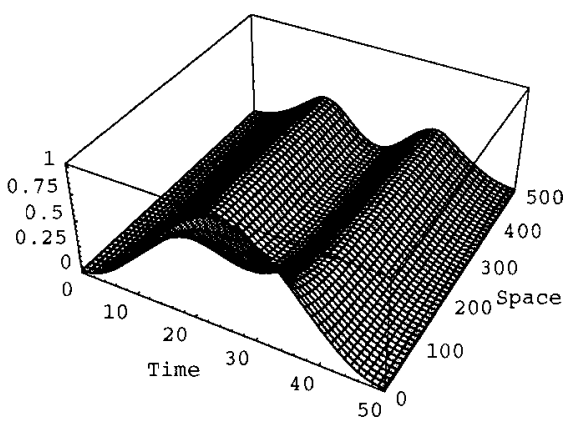

(b)

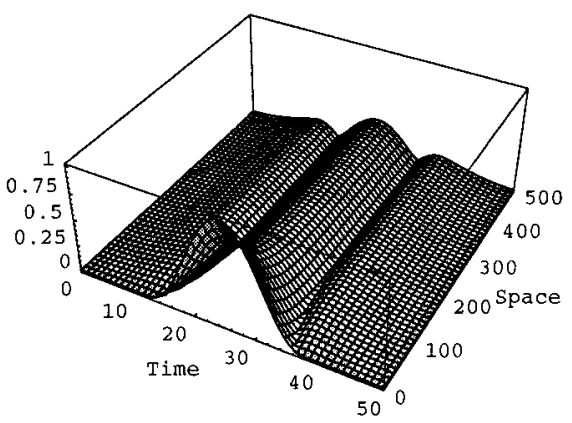

(c)

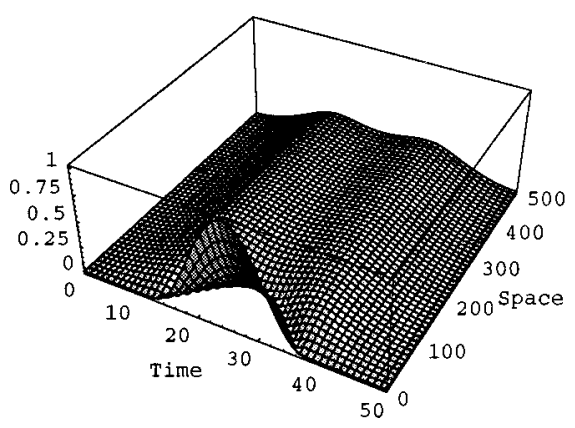

FIG. 7. Normalized field amplitudes $\left|\Omega_{0}(\zeta, \tau)\right| / \Omega_{0} \quad$ (a), $\left|\Omega_{1}(\zeta, \tau)\right| / \Omega_{1}$ (b), $\left|\Omega_{2}(\zeta, \tau)\right| / \Omega_{2}$ (c), for initial pulses $\Omega_{0}(0, \tau)$ $=\Omega_{0} \sin ^{2}\left(\tau \pi / \tau_{p}\right)\left(0 \leqslant \tau \leqslant \tau_{p}\right), \Omega_{1}(0, \tau)=\Omega_{1} \sin ^{2}\left[2\left(\tau-\tau_{p} / 4\right) \pi / \tau_{p}\right]$ $\left(\tau_{p} / 4 \leqslant \tau \leqslant 3 \tau_{p} / 4\right), \Omega_{2}(0, \tau)=\Omega_{2} \sin ^{2}\left[2\left(\tau-\tau_{p} / 4\right) \pi / \tau_{p}\right]\left(\tau_{p} / 4 \leqslant \tau\right.$ $\left.\leqslant 3 \tau_{p} / 4\right)$. The system is initially in the superposition $|\psi(\zeta, 0)\rangle$ $=1 / \sqrt{3}|0\rangle+1 / \sqrt{6}|1\rangle+1 / \sqrt{2}|2\rangle$ and $\Omega_{0}=\Omega_{1}=\Omega_{2}=0.1$. The remaining medium and pulse parameters are the same as in Fig. 2.

$$
\begin{aligned}
& \Omega_{1}(\zeta, \tau)=\frac{1}{\lambda} \alpha^{*} \beta \kappa_{1}^{\prime}\left(-1+e^{-i \lambda \zeta}\right) \bar{\Omega}(\tau), \\
& \Omega_{2}(\zeta, \tau)=\frac{1}{\lambda} \alpha^{*} \gamma \kappa_{2}^{\prime}\left(-1+e^{-i \lambda \zeta}\right) \bar{\Omega}(\tau) .
\end{aligned}
$$

Therefore, via a coherent internal generation process, a medium coherently prepared in three of the lower levels will generate two additional fields from the applied field. This will occur only if the initial state is a coherent superposition. In particular, from the right-hand side term of Eqs. (29) and (30) it can be seen that the Rabi frequency of a generated pulse on a specific transition is proportional to the initial coherence of the corresponding Raman transition with the injected field. (a)

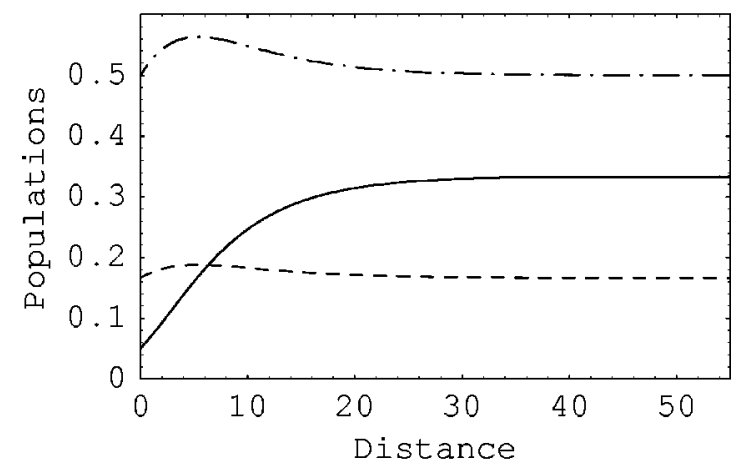

(b)

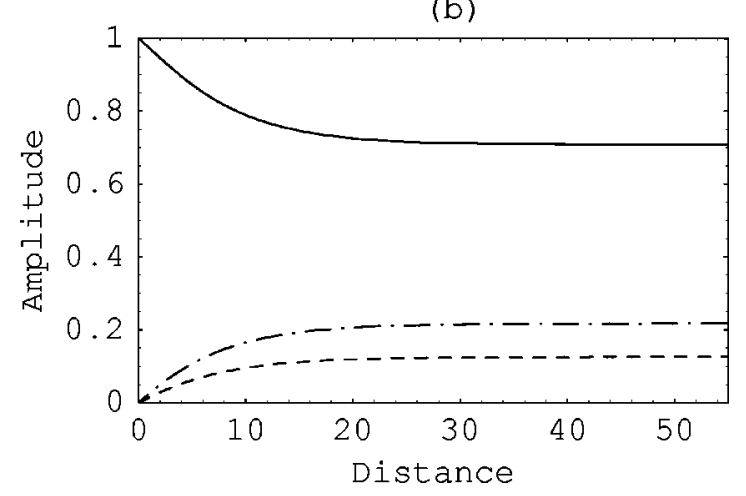

FIG. 8. In the upper figure, we present the populations of states $|0\rangle$ (solid curve), $|1\rangle$ (dashed curve), and $|2\rangle$ (dot-dashed curve) as a function of distance at $\tau=60$. In the lower figure, we present the maximum of the normalized field amplitudes as a function of distance: $\left|\Omega_{0}(\zeta, \tau)\right| / \Omega_{0}$ (solid curve), $\left|\Omega_{1}(\zeta, \tau)\right| / \Omega_{0}$ (dashed curve), and $\left|\Omega_{2}(\zeta, \tau)\right| / \Omega_{0}$ (dot-dashed curve). The results have been obtained by numerically solving Eqs. (8) and (9). The initial pulse is $\Omega_{0}(0, \tau)=\Omega_{0} \sin ^{2}\left(\tau \pi / \tau_{p}\right)\left(0 \leqslant \tau \leqslant \tau_{p}\right) \quad$ and $\quad \Omega_{1}(0, \tau)=\Omega_{2}(0, \tau)=0$. The system is initially in the superposition $|\psi(\zeta, 0)\rangle=1 / \sqrt{3}|0\rangle$ $+1 / \sqrt{6}|1\rangle+1 / \sqrt{2}|2\rangle$ and $\gamma=10$. The remaining medium and pulse parameters are the same as in Fig. 2.

In order to simplify the form of the above equations we assume, as in the previous section, that the propagation constants are equal. Setting $a_{0}=a_{1}=a_{2}=a$ leads to $\kappa_{0}^{\prime}=\kappa_{1}^{\prime}$ $=\kappa_{2}^{\prime}=\kappa$. The pulses that describe the parametric generation process, Eqs. (28)-(30) then reduce to

$$
\begin{gathered}
\Omega_{0}(\zeta, \tau)=\left(|\alpha|^{2} e^{-i \kappa \zeta}+|\beta|^{2}+|\gamma|^{2}\right) \bar{\Omega}(\tau), \\
\Omega_{1}(\zeta, \tau)=\alpha^{*} \beta\left(-1+e^{-i \kappa \zeta}\right) \bar{\Omega}(\tau), \\
\Omega_{2}(\zeta, \tau)=\alpha^{*} \gamma\left(-1+e^{-i \kappa \zeta}\right) \bar{\Omega}(\tau) .
\end{gathered}
$$

Parametric generation of two fields by a single injecting field is illustrated in Fig. 5, where the time and space evolution of the laser pulses is shown. The results have been obtained using Eqs. (31)-(33). Note that the chosen parameters lead to the incoming and generated fields being matched, i.e., they obtain the envelope of the injected field in the medium. This follows directly from Eqs. (31)-(33). The accuracy of the analytical results has been assessed by comparing with 
the numerical solution of Eqs. (8) and (9). As can be seen in Fig. 6, both results are once again in very good agreement.

We now consider the propagation dynamics of three injected pulses, with the duration of one of the pulses being twice as long as the other two pulses. As illustrated in Fig. 7, in contrast to the case of parametric generation, the pulses in the medium evolve with completely different envelopes. Matched pulses are not created. The origin of this behavior will be explained in the following section.

\section{PULSE PROPAGATION AT LARGE DISTANCES: A GENERAL RELATION}

In this section, let us again assume that the system is initially prepared in a coherent superposition of all of the lower states according to Eq. (20). However, the assumption that the laser-matter interaction is weak will be relaxed. Instead, we postulate that the Rabi frequencies $\Omega_{n}(\zeta, \tau)$ become independent of $\zeta$ as $\zeta \rightarrow \infty$, i.e.,

$$
\frac{\partial}{\partial \zeta} \Omega_{n}(\zeta, \tau)=0, \quad n=0,1,2
$$

We note that in the weak-field regime, Eqs. (24)-(26) imply that this occurs when $\zeta \gg \bar{\zeta}$. When Eqs. (34) are satisfied, from Eqs. (9), it is seen that $b_{3}(\zeta \rightarrow \infty, \tau)=0$. This, in turn, implies that Eq. (8) reduces to

$$
\frac{\partial}{\partial \tau} b_{n}(\zeta \rightarrow \infty, \tau)=0,
$$

with $n=0,1,2$, and therefore

$$
b_{0}(\zeta \rightarrow \infty, \tau)=\alpha, \quad b_{1}(\zeta \rightarrow \infty, \tau)=\beta, \quad b_{2}(\zeta \rightarrow \infty, \tau)=\gamma .
$$

Using the expression for $(\partial / \partial \tau) b_{3}(\zeta, \tau)$ as given by Eq. (8), the following general expression relating the initial probability amplitudes with the Rabi frequencies $\Omega_{n}(\zeta, \tau)$ at long distances is obtained

$$
\alpha^{*} \Omega_{0}(\zeta \rightarrow \infty, \tau)+\beta^{*} \Omega_{1}(\zeta \rightarrow \infty, \tau)+\gamma^{*} \Omega_{2}(\zeta \rightarrow \infty, \tau)=0 .
$$

This result shows that, irrespective of the strength of the laser-matter interaction (within the constrains of the rotatingwave approximation) the laser fields in a coherently prepared medium are not absorbed by the medium but are reshaped in order to fulfill the general relation given by Eq. (37). This phenomenon is illustrated in Fig. 8, where the atomic populations at the end of the pulse and the maximum field amplitudes are shown as a function of the distance in the medium. The results were obtained by numerically solving Eqs. (8) and (9). It is seen that at large distances, the populations and the field amplitudes become constant, thereby justifying Eq. (34).

It is straightforward to show that the analytical solutions given in Sec. IV, which are valid for weak laser-matter interactions, satisfy Eq. (37). As noted in the previous section, the propagation of laser pulses in a coherently prepared tripod scheme does not necessarily lead to the creation of matched pulses, and this is confirmed by Eq. (37).

\section{CONCLUSIONS}

The potential for using resonant nonlinear optics to engineer the optical response of a medium has been recognized for a number of years now. Recent work on the storing of laser pulses, or more specifically the information they carry, in optically active media $[24,25]$ has provided a remarkable example of this potential. These resonant nonlinear optical processes rely on the creation of a coherence in the medium by one pulse, which in turn can strongly modify absorption and dispersion of a second pulse. In this paper, we have investigated resonant nonlinear optical processes that occur when a medium is initially prepared in a coherent superposition. In particular, for a tripod system we have demonstrated, both analytically and numerically, that parametric pulse generation is possible, and have identified conditions that lead to either complete absorption by the medium or complete transparency. We have also shown that at distances large compared to the three-photon Beer's length, the propagation of the pulses becomes independent of distance. The pulse amplitudes then depend only on time and, in particular, must satisfy a simple algebraic constraint involving only the three Rabi frequencies, or pulse amplitudes, and initial level amplitudes, respectively.

\section{ACKNOWLEDGMENTS}

E.P. would like to thank Z. Kis for useful discussions. This work was supported by the UK Engineering and Physical Sciences Research Council and by the European Community's Human Potential Program under Contract No. HPRNCT-1999-00129, COCOMO.
[1] S. E. Harris, Phys. Today 50 (7), 37 (1997).

[2] J. P. Marangos, J. Mod. Opt. 45, 471 (1998).

[3] M. D. Lukin, P. R. Hemmer, and M. O. Scully, Adv. At., Mol., Opt. Phys. 42, 347 (2000).

[4] Z. Ficek and S. Swain, LANL e-print quant-ph/0109100, J. Mod. Opt. (to be published).

[5] M. O. Scully, Phys. Rev. Lett. 67, 1855 (1991).

[6] S. E. Harris, Phys. Rev. Lett. 70, 552 (1993).

[7] M. Fleischhauer and T. Richter, Phys. Rev. A 51, 2430 (1995).
[8] A. Rahman, R. Grobe, and J. H. Eberly, in Coherence and Quantum Optics VII, edited by J. H. Eberly, L. Mandel, and E. Wolf (Plenum Press, New York, 1996), p. 449.

[9] J. H. Eberly, A. Rahman, and R. Grobe, Phys. Rev. Lett. 76, 3687 (1996).

[10] G. Vemuri, K. V. Vasavada, G. S. Agarwal, and Q. Zhang, Phys. Rev. A 54, 3394 (1996).

[11] E. Cerboneschi and E. Arimondo, Phys. Rev. A 54, 5400 (1996). 
[12] V. V. Koslov and J. H. Eberly, Opt. Commun. 179, 85 (2000).

[13] S. E. Harris and M. Jain, Opt. Lett. 22, 636 (1997).

[14] W. Harshawardan and G. S. Agarwal, Phys. Rev. A 58, 598 (1998)

[15] L. Deng, M. G. Payne, and W. R. Garrett, Phys. Rev. A 58, 707 (1998).

[16] M. D. Lukin, P. R. Hemmer, M. Löffler, and M. O. Scully, Phys. Rev. Lett. 81, 2675 (1998).

[17] S. H. Choi and G. Vemuri, Opt. Commun. 153, 257 (1998).

[18] M. Jain, H. Xia, G. Y. Yin, A. J. Merriam, and S. E. Harris, Phys. Rev. Lett. 77, 4326 (1996).

[19] A. S. Zibrov, M. D. Lukin, and M. O. Scully, Phys. Rev. Lett. 83, 4049 (1999).

[20] A. J. Merriam, S. J. Sharpe, M. Shverdin, D. Manuszak, G. Y. Yin, and S. E. Harris, Phys. Rev. Lett. 84, 5308 (2000).

[21] A. V. Sokolov, D. R. Walker, D. D. Yanuz, G. Y. Yin, and S. E. Harris, Phys. Rev. Lett. 85, 562 (2000).

[22] J. Q. Liang, M. Katsuragawa, F. Le Kien, and K. Hakuta, Phys. Rev. Lett. 85, 2474 (2000).

[23] A. F. Huss, N. Peer, R. Lammegger, E. A. Korsunsky, and L. Windholz, Phys. Rev. A 63, 013802 (2001).
[24] C. Liu, Z. Dutton, C. H. Behroozi, and L. V. Hau, Nature (London) 409, 490 (2001).

[25] D. F. Phillips, A. Fleischhauer, A. Mair, R. L. Walsworth, and M. D. Lukin, Phys. Rev. Lett. 86, 783 (2001).

[26] R. G. Unanyan, M. Fleischhauer, B. W. Shore, and K. Bergmann, Opt. Commun. 155, 144 (1998); H. Theuer, R. G. Unanyan, C. Habscheid, K. Klein, and K. Bergmann, Opt. Express 4, 77 (1999).

[27] Z. Kis and S. Stenholm, Phys. Rev. A 64, 063406 (2001).

[28] R. G. Unanyan, B. W. Shore, and K. Bergmann, Phys. Rev. A 59, 2910 (1999).

[29] Z. Kis and F. Renzoni Phys. Rev. A 65, 032318 (2002).

[30] B. S. Ham and P. R. Hemmer, Phys. Rev. Lett. 84, 4080 (2000).

[31] B. S. Ham, Appl. Phys. Lett. 78, 3382 (2001).

[32] E. Paspalakis and P. L. Knight, J. Mod. Opt. 49, 87 (2002).

[33] Traditionally, matched pulses refer to pulses that have the same Rabi frequencies [6-10]. However, in the present paper we will be concerned with the modulus of the pulses and will refer to pulses that have magnitudes that are proportional as being matched. 